\title{
ELM mitigation with pellet ELM triggering and implications for PFCs and plasma performance in ITER*
}

\author{
L.R. Baylor ${ }^{a}$, P.T. Lang ${ }^{b}$, S. L. Allen ${ }^{c}$, S.K. Combs ${ }^{a}$, N. Commaux ${ }^{a}$, T.E. Evans ${ }^{d}$, \\ M.E. Fenstermacher ${ }^{\mathrm{c}}$, G. Huijsmans ${ }^{\mathrm{e}}$, T.C. Jernigan ${ }^{\mathrm{a}}$, C.J. Lasnier ${ }^{\mathrm{c}}$, A.W. Leonard ${ }^{\mathrm{d}}$, A.

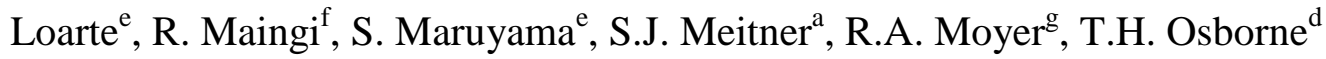 \\ ${ }^{a}$ Oak Ridge National Laboratory, PO Box 2008, Oak Ridge, Tennessee 37830-8050, USA \\ ${ }^{b}$ Max Plank Institute für Plasmaphysik, EURATOM Association., Boltzmannstr. 2, 85748 Garching, \\ Germany \\ ${ }^{c}$ Lawrence Livermore National Laboratory, 700 East Ave, Livermore, California 94550, USA \\ ${ }^{d}$ General Atomics, PO Box 85608, San Diego, California 92186-5608, USA \\ ${ }^{e}$ ITER Organization, CS 90 046, 13067 St. Paul lez Durance Cedex, France \\ ${ }^{f}$ Princeton Plasma Physics Laboratory, Princeton, New Jersey, USA \\ ${ }^{g}$ University of California San Diego, 9500 Gilman Dr., La Jolla, California 92093-0417, USA
}

\begin{abstract}
The triggering of rapid small edge localized modes (ELMs) by high frequency pellet injection has been proposed as a method to prevent large naturally occurring ELMs that can erode the ITER plasma facing components (PFCs). Deuterium pellet injection has been used to successfully demonstrate the on-demand triggering of edge localized modes
\end{abstract}

\footnotetext{
* This work was supported by the Oak Ridge National Laboratory managed by UT-Battelle, LLC for the US Department of Energy under DE-AC05-00OR22725 and the US Department of Energy under DEFC02-04ER54698, DE-AC52-07NA27344, and DE-FG02-07ER54917. The views and opinions expressed herein do not necessarily reflect those of the ITER Organization.

PACS: 52.55.Fa, 52.55.-s, 28.52.Cx

JNM keywords: Plasma properties, Gases

PSI-21 keywords: Gas injection \& Fueling; Pellet; DIII-D; ELM

\# Corresponding author address: ORNL Box 2008, Oak Ridge, TN $37831-6169$, USA.

\# Corresponding author E-mail: BaylorLR@ornl.gov

Presenting author: Larry R. Baylor
} 
(ELMs) at much higher rates and with much smaller intensity than natural ELMs. The proposed hypothesis for the triggering mechanism of ELMs by pellets is the local pressure perturbation resulting from reheating of the pellet cloud that can exceed the local high-n ballooning mode threshold where the pellet is injected. Nonlinear MHD simulations of the pellet ELM triggering show destabilization of high-n ballooning modes by such a local pressure perturbation.

A review of the recent pellet ELM triggering results from ASDEX Upgrade (AUG), DIII-D, and JET reveals that a number of uncertainties about this ELM mitigation technique still remain. These include the heat flux impact pattern on the divertor and wall from pellet triggered and natural ELMs, the necessary pellet size and injection location to reliably trigger ELMs, and the level of fueling to be expected from ELM triggering pellets and synergy with larger fueling pellets. The implications of these issues for pellet ELM mitigation in ITER and its impact on the PFCs are presented along with the design features of the pellet injection system for ITER. 


\section{Introduction}

The H-mode confinement regime of plasma operation is planned for ITER in order to achieve high fusion performance. It is characterized by a steep pressure gradient and "pedestal" at the plasma edge that is expected to lead to the quasi-periodic instability of edge-localized modes (ELMs).[1] ELMs expel periodic bursts of particles and energy from the plasma, which if large enough can pose a serious threat to the PFCs by erosion and melting from the high heat fluxes and to the plasma burn by producing a source of impurities into the plasma. The intensity of ELMs in ITER is expected to be quite large with a relatively small impact area on the divertor and therefore must be controlled to tolerable levels in order to achieve sufficient lifetime of the PFCs [2].

This paper describes the progress of using small deuterium pellets to control the ELM size by on-demand triggering of ELMs at rates much higher than the natural Type I ELM frequency to reduce the ELM intensity [3]. This technique known as pellet ELM pacing has been proposed as a method to prevent large ELMs that can damage the ITER divertor surface.[4] Key questions that remain to be addressed for this technique are the level of ELM divertor heat flux reduction achievable at frequencies of 10-60 times the natural ELM frequency as is needed for ITER [2] and the area and level of toroidal asymmetry in the divertor heat flux for small pellet triggered ELMs.

In Sec. 2 of this paper we describe the process of the pellet ELM triggering phenomenon. In Sec. 3 the details of the ELM triggering and pacing results on different machines are reviewed and aspects of the resulting heat flux from pellet induced ELMs are described. In Sec. 4 we discuss the implications of this work for ITER, in particular 
how the triggering of ELMs could be utilized to extend PFC lifetimes. Finally, in Sec. 5 we present a summary of our main conclusions from this work.

\section{Pellet ELM Triggering Phenomenon}

A working hypothesis on the mechanism for pellets to trigger an ELM has been formulated and has been compared with experiments and modeling. In this hypothesis the ablation of the pellets produces a local density increase on flux tubes that the pellet crosses as illustrated in Fig. 1. At each flux tube where a pellet cloud is released by the pellet the density increase is proportional to the ablation rate at that location. The local temperature at the location of the cloud is reduced because of the adiabatic nature of the ablation process. Once released from the pellet, that density cloud expands along the field line of the flux tube at the sound speed of the ions. Simultaneously with this expansion, the cloud is being heated by electrons along the field lines at a much higher speed dictated by the parallel thermal diffusion. This results in a local pressure cloud formation that expands along the field line. This pressure cloud leads to strong pressure gradients along and especially perpendicular to the field lines. When this pressure gradient exceeds a threshold, a local high-n ballooning mode is excited that grows non-linearly until an ELM crash is produced. Because of the local nature of this ballooning mode it is likely that such ELMs are of very high $n$ mode and different from moderate $n$ natural ELMs from peeling-ballooning modes that are more axisymmetric in nature.

On DIII-D, tangential viewing fast camera images of low field side (LFS) midplane and divertor injected pellets have shown a single plasma filament becomes highly visible just in front of the pellet ablation cloud as shown in Fig. 1 of Ref [3], similar to that observed previously for vertically injected pellets. These filament structures from the 
LFS injected pellets have been observed to strike the outer vessel wall near the injection location within $200 \mu$ s of their formation. This is consistent with the hypothesis that the pellet cloud produces a local pressure perturbation that triggers a local ballooning mode instability that manifests itself as the reduced size ELM filament that accelerates across the scrape-off layer toward the wall. Similar observations have been made on JET with a fast framing camera showing a regular footprint on the wall exists with pellet triggered ELMs compared to a random wall interaction from natural ELMs $[5,6]$.

Recent data taken with a tangential viewing infrared camera on DIII-D has shown that there is some local heating of the wall near the pellet injection locations when an ELM is triggered by a pellet. An example of these measurements is shown in Fig. 2 for a LFS midplane injected pellet that triggered a large ELM, nearly the size of a natural ELM. Poloidal line profiles of the heat flux have been determined from different frames of the IR camera images. The image frame taken just after a LFS midplane pellet was injected shows a noticeably higher heat flux than from frames taken after a natural ELM or after an ELM triggered by a pellet that was injected near the LFS lower divertor, labeled R-2. The magnitude of the wall heat flux is less than $20 \%$ of the divertor heat flux at that poloidal angle. The IR camera also measures a heat flux near the R-2 injection port only when a pellet from that port triggers an ELM and then some heat flux is also detected on the inner wall near the X-point. This is consistent with fast framing camera movies of the plasma that show the separatrix moves inward when an ELM is triggered. When small pellets enter the plasma from these ports that do not trigger an ELM, there is no heat flux signature observed on the wall at these locations. This result is also consistent with the visible camera observations on JET of filamentary structures striking 
the wall near the LFS midplane injection location when pellets from there triggered ELMs [6].

From the hypothesis of a high-n ballooning mode triggered by the pellet during the ablation process one can calculate the expected strike points of the field lines of a single released ELM filament on the divertor. Using the field line integration code TRIP3D [7] we have calculated the footprint of a single filament of flux that has a $1 \mathrm{~cm}$ diameter for a pellet injected from the DIII-D LFS midplane as that filament moves in the scrape-off layer in the major radius direction toward the outer wall. This motion is expected from a polarization drift effect in the cloud similar to blobs in the scrape-off layer and pellet deposition physics [8,9]. We see that the footprint in the divertor floor is not axisymmetric and as the filament reaches a position near the wall the footprint moves significantly away from the normal outer strike point. One would expect most of the heat in this flux tube to be exhausted to the divertor well before the filament reaches the wall [10], thus the heat flux pattern would not necessarily show this much radial expansion.

Measurements in JET by Wenninger et al [11] have shown that a toroidally asymmetric divertor power deposition often exists from ELMs triggered by large fueling pellets injected from the LFS midplane. A strong secondary heat flux impact point is observed that extends up to $5 \mathrm{~cm}$ outside of the normal strike point and with an intensity as high or higher than the natural ELM strike point power density. The strike point locations from these fairly large ELMs was also found to vary as a function of the toroidal magnetic field, which is consistent with field line mapping of a filament like structure in the scrape-off layer moving in the major radius direction [11]. No such structure has been observed in the DIII-D pellet induced ELMs with small pellets injected 
from any location, even when triggering rather large ELMs. The divertor viewing location is however rather close toroidally to the pellet injection location where strike point splitting from the filament field line mapping is not predicted to be strong.

In AUG with carbon walls (AUG-C) [12] it was reported that the natural and pellet triggered ELMs of the same frequency were of the same intensity and did not demonstrate any significant difference in the radial heat flux profile in either the inner or outer strike points. The pellets in this case were injected from the vertical high field side (HFS). The hypothesis there was that the pellet induced perturbation had spread toroidally before reaching the LFS and therefore no occurrences of stripes related to the toroidal angle were observed. Similar results were reported later from AUG-C with divertor cooling by Ar seeding [12] and more recently with the all metal wall configuration [13].

In recent results from JET and AUG with metal wall configurations (JET-ILW and AUG-W) it was discovered that if pellets are injected too soon after an ELM, no ELM is triggered independent of the pellet perturbation size. The effect known as a trigger lag time was not present in either machine when they were configured with carbon walls. The use of nitrogen seeding of the divertor in AUG-W has reduced this lag time effect significantly from $\sim 10 \mathrm{~ms}$ to approximately $2 \mathrm{~ms}$. On the other hand, lag times of up to $90 \mathrm{~ms}$ were observed in experiments investigating the ITER base line scenario. The cause of this lag time is not yet understood and is presumably due to differences in the pedestal dynamics without the carbon impurity present. 


\section{Pellet ELM Pacing Investigations}

The principle of pellet ELM pacing has been demonstrated to various levels on different machines [AUG, DIII-D, JET]. These results while encouraging lead to a number of questions to be resolved to be able to successfully employ this technique on ITER to extend the divertor lifetime. Some of these key questions are what size and speed of pellet is required to trigger an ELM reliably and where in the plasma must it penetrate, what is the triggered ELM divertor heat flux footprint and how does it scale with ELM size, does the divertor heat flux follow a $1 / \mathrm{f}_{\mathrm{ELM}}$ scaling as the ELM frequency multiplier reaches the level needed for ITER, and is there synergy between the pellet ELM triggering and fueling pellets injected from the HFS.

Experiments on JET-ILW and DIII-D using low field side pellet injection have been analyzed to determine the range of pellet sizes and speed needed to trigger ELMs for a given plasma condition. The JET results for a set of small LFS midplane injected pellets were documented in Fig. 7 of Ref. [14] and shown in Fig.3a. Pellets greater in size than $2 \times 10^{20}$ atoms ( $\sim 4 \%$ of the plasma inventory as measured at the injector) and a speed of $100 \mathrm{~m} / \mathrm{s}$ were $100 \%$ reliable at triggering an ELM after at least $20 \mathrm{~ms}$ following a previous ELM event. Recently analyzed results from DIII-D are shown in Fig. 3b where LFS midplane injected pellets are plotted as a function of size and speed. These were all injected in similar ITER shape discharges with the same normalized $\beta$ of $\sim 1.8$. There is not a significant dependence on pellet speed whether or not an ELM is triggered. The main dependence is on pellet size, where a full size pellet is a cylinder of $1.3 \mathrm{~mm}$ diameter and $0.9 \mathrm{~mm}$ length ( $7 \times 10^{19}$ atoms, $\sim 1 \%$ of plasma inventory). Pellets at least half this size (as measured by a microwave cavity just before entering the plasma) were 
found to trigger an ELM independent of the time since a previous ELM. The edge pedestal pressure varies through an ELM cycle and so it is surprising to some extent that this necessary size perturbation does not appear to be a strong function of the pedestal pressure.

A key aspect in the level of ELM control that is needed for ITER is the area over which the ELM energy is deposited, the so called heat flux footprint or wetted area (AELM) [15]. On JET, the Type-I ELM wetted area was found to scale weakly with the total ELM energy deposited $\mathrm{E}$ as $\mathrm{E}^{0.22}$ [16]. The outer divertor leg heat flux profile from pellet induced small ELMs on DIII-D has been compared with the large natural ELMs [17] that occur in the ITER like scenario. It was found that the small pellet triggered ELM wetted area $A_{\text {ELM }}$ was $~ 115 \%$ of the inter-ELM wetted area and was a factor of $\sim 2$ smaller than from the large natural ELMs. This decrease in $\mathrm{A}_{\mathrm{ELM}}$ is consistent with the earlier JET scaling with ELM energy loss. Fig. 4 shows a case where the small pellet induced ELM heat flux profile is overlaid with the natural ELM heat flux and multiplied by a factor of 7.5 to make the profiles more easily comparable. It is clear that the natural ELM has a larger wetted area by nearly a factor of 2 from the pellet induced and interELM heat flux profiles. The pellet triggered ELMs do not demonstrate any structure in the heat flux profile outside of the usual strike point. As mentioned before, a structure in the outer leg heat flux unique to pellet induced ELMs has been observed on JET that has not been observed on AUG or DIII-D. Heat flux measured at the outer wall near the injection location of pellets is small compared to the divertor heat flux.

The question remains as to how well the ELM peak heat flux in the divertor follows the $1 / f_{\text {ELM }}$ scaling that is anticipated for reduced ELM sizes. The average peak heat 
fluxes from pellet triggered ELMs on DIII-D showed a trend of reduced levels as a function of the pellet frequency proportional to $1 / f_{\text {pell }}[18]$. The peak heat flux on the outer strike point was found to be larger than on the inner strike point for all ELM frequencies including the $5 \mathrm{~Hz}$ natural ELMs. JET-ILW results with LFS fueling size pellets were recently presented showing that for a scenario where the ELM frequency was increased by about a factor of 2 that there was very little change in the ELM peak heat flux. [14]. However these ELMs on JET triggered by the large pellets remain quite large, nearly $5 \%$ of the total plasma stored energy is expelled in each ELM.

\section{Implications for ITER}

An evaluation of the limits of ELM induced heat flux on the operation of ITER have been carried out and indicate that uncontrolled ELMs will greatly exceed the heat flux on the $\mathrm{W}$ divertor that would result in localized melting [2]. Using the wetted area scaling of the divertor heat flux with ELM size, largely based on JET results, implies that an ELM frequency increase of a factor of 45 is needed for ITER, where the natural ELM frequency is predicted to reach $\sim 1 \mathrm{~Hz}$ at the full $15 \mathrm{MA}$ operating plasma current as shown in Fig. 5. This assumes that the ELM heat flux intensity is inversely proportional to the ELM frequency and that the wetted area scales with ELM energy deposited E as $\mathrm{E}^{0.22}$. As mentioned in the previous section, the DIII-D ELM wetted area measurements from large natural and small pellet triggered ELMs are in qualitative agreement with this JET scaling. Therefore none of the present experiments have shown any indication that the predicted needs for the paced ELM rate will not be less due to a larger than expected 
ELM wetted area. There is some indication from JET that the pellet triggered ELMs can have some toroidal asymmetry in the heat flux pattern on the divertor. In principle on ITER this can be somewhat compensated by the simultaneous use of 3 different toroidal injection locations for the pellets to help spread any locally induced heat fluxes around the machine toroidally [19].

The primary fueling mechanism for ITER during the DT burn phase campaign is anticipated to be from DT pellets injected from the HFS (inner wall). These pellets are planned to be in the $5.5 \mathrm{~mm}$ cylindrical size range with a speed up to $300 \mathrm{~m} / \mathrm{s}$ [19] at a 1$4 \mathrm{~Hz}$ injection rate. It is well known that HFS injected pellets are able to trigger ELMs as observed on AUG, DIII-D and JET, however this rate of injection from the fueling pellets is well below that needed for ELM mitigation. The question remains whether the ELM size minimization from the high rate of small pellets is compatible with the infrequent larger size pellets simultaneously being used to fuel the plasma. Some attempt to examine this have been made on DIII-D where $1.3 \mathrm{~mm}$ pellets were injected from the inner wall in the same shot as $30 \mathrm{~Hz}$ smaller pellets were injected from the LFS divertor location in ITER shaped plasmas at $\beta_{\mathrm{N}}$ of 1.8 [17]. The HFS pellets did indeed trigger ELMs that were not any larger in size than those from the LFS pellets. There was noticeable direct fueling from the HFS pellets while none was observed from the LFS pellets as can be seen in Fig. 6. No significant reduction in plasma confinement was observed when the HFS pellets were applied. While in principle this looks favorable for ITER, it is difficult to mimic the expected HFS pellet penetration on present machines with currently available pellet sizes, which penetrate further in minor radius than expected on ITER. 
The important issue on the required pellet size to reliably trigger ELMs (which has implications for the fuel throughput and divertor pumping needed) in the ITER pellet injection geometry (in the low field side of the plasma above the X-point), and possible toroidal asymmetries of divertor heat flux from pellet triggered ELMs are being addressed by modeling and comparison with experiment. Modeling of ELM triggering by pellet injection has been performed with the nonlinear MHD code JOREK, which has been upgraded to include an appropriate description of the pellet ablation physics [18]. Predictions have been compared with experiments in DIII-D in which ELMs have been triggered by injection of pellets at the LFS midplane and X-point locations. The JOREK simulations show that triggering of ELMs by pellet injection is caused by the large toroidally-localized edge pressure gradients produced by the ablating pellet. When these gradients exceed a threshold, ballooning modes grow nonlinearly leading to the ELM crash. Exceeding this pressure gradient threshold implies that, for given pedestal plasma conditions, a minimum pellet size is required to trigger an ELM, which is in agreement with the measurements shown on JET and DIII-D. JOREK modeling shows that this minimum pellet size depends on the injection geometry and that for injection near the LFS X-point, the required pellet size in DIII-D is a factor of $\sim 2$ smaller than for midplane injection. This implies that the ITER pellet injection geometry for ELM triggering [19] is advantageous to reduce the fuel throughput requirements associated with this ELM control scheme.

Modeling of the divertor power flux for pellet triggered ELMs in DIII-D predicts a sizeable asymmetric secondary peak (i.e. of similar magnitude to the primary separatrix strike point) about $3 \mathrm{~cm}$ outward from the primary peak. This asymmetry is strongest at a 
toroidal location 180 degrees away from the injection location, while the divertor heat flux measurements are made only $\sim 45$ degrees from the injection locations. This predicted splitting of the heat flux pattern away from the injection location effectively reduces the peak heat flux by a factor of 2 , so in principle it may be a favorable effect. This is similar to the effect that was observed on JET [11] and is predicted for ITER [18]. Another scheme that can be employed simultaneously with the pellets injected from 3 injection locations to help spread the ELM heat flux on the divertor is to oscillate the strike point in and out during a plasma pulse. It remains to be seen how effective this will be at mitigating divertor material melt damage.

The present experiments carried out on DIII-D and JET are as much as a factor of 5 away from the ELM frequency multiplier anticipated for ITER and the number of ELMs needed per energy confinement time to mitigate the heat flux, which could be as much as 200, are a factor of 10 higher than achieved. In addition, the plasma conditions of ITER operating near the density limit with a scrape off layer that is opaque to neutral penetration is quite different from today's experiments. These differences make it difficult to predict with any certainty how ITER will respond to pellet ELM triggering at the rates that are anticipated. Future experiments will try to reach higher ELM frequencies, but plasma conditions will necessarily remain somewhat different than in ITER. Because of these uncertainties, the pellet injection system for ITER is being design with flexibility in pellet size, injection rate, and injection location to be able to optimize the performance as necessary [20]. The nominal ELM triggering pellet size is $1.5 \%$ of the nominal plasma inventory with an adjustment ability of $+/-0.5 \%$ at a rate of $16 \mathrm{~Hz}$ per injector with up to 6 injectors eventually available. 3 different toroidal positons are 
available for injection, each with 2 HFS inner wall locations and one LFS location near the divertor.

\section{SUMMARY AND CONCLUSIONS}

In summary, experiments have shown that a large increase in ELM frequency approaching that needed for ITER is possible with corresponding reductions in ELM energy loss using the on-demand triggering of ELMs by the injection of small pellets. Both the inner and outer legs of the divertor are found to have a reduced peak heat flux when suitable size pellets and injection location are employed. Experimental research and modeling are being undertaken around the world with much progress being made to better understand the ELM triggering mechanism and its predicted effects in ITER.

The designed ITER pellet injection scheme seems well planned for the injection location to minimize the ELM triggering fueling and the number of injection locations is favorable for redundancy and the spread of any toroidal asymmetric heat fluxes. The synergy of pellet fueling with large DT inner wall injected pellets with ELM mitigation techniques remains a key issue that may not be fully addressed until ITER begins operation.

\section{ACKNOWLEDGMENTS}

This work was supported by the Oak Ridge National Laboratory managed by UTBattelle, LLC for the US Department of Energy under DE-AC05-00OR22725 and the US Department of Energy under DE-FC02-04ER54698, DE-AC52-07NA27344, and DEFG02-07ER54917. The views and opinions expressed herein do not necessarily reflect those of the ITER Organization. 


\section{References}

[1] H. Zohm, Plasma Phys. Controlled Fusion 38, (1996) 105.

[2] A. Loarte et al., Nucl. Fusion 54(2014) 033007.

[3] L.R. Baylor et al., Phys. Rev. Lett. (2013) 245001.

[4] P. T. Lang, et al., Nucl. Fusion 44, (2004) 665.

[5] G. Kocsis, et al. 37th EPS Conf. on Plasma Physics, Dublin, Ireland 2010 Europhysics Conference Abstracts, Vol. 34A, P-4.136

[6] P.T. Lang, et al., Nucl. Fusion 51, (2011) 033010.

[7] T.E. Evans, et al., Phys. Plasmas 9 (2002) 4957.

[8 ] O.E. Garcia, Plasma Fus. Res. 4 (2009) 019.

[9 ] V.A. Rozhansky, et al., Plasma Phys. Control. Fusion 37, (1995) 399.

[10] J. A. Boedo, et al., Phys. Plasmas 12, (2005) 072516.

[11] R. P. Wenninger, et al., Nucl. Fusion 53, (2011) 105002.

[12] P. T. Lang, et al., Nucl. Fusion 48, (2008) 095007.

[13] P.T. Lang et al., to be published in Nucl. Fusion.

[14] P.T. Lang, et al, Nucl. Fusion 53 (2013) 043004.

[15] T.H. Eich, et al., J. Nucl. Mat. 415 (2011) S856.

[16] W. Fundamenski, et al., Nucl. Fus. 51 (2011) 083028.

[17] L.R. Baylor, et al., Phys. Plasmas 20, 082513 (2013).

[18] S. Futatani, et al., Nucl. Fusion 54 (2014) 073008. 
[19] S.K. Combs, et al., Fus. Eng. Design 87 (2012) 534.

[20] L.R. Baylor, et al., Nucl. Fusion 47 (2007) 1598.

\section{LIST OF FIGURE CAPTIONS}

FIG. 1. Pellet cloud geometry showing expansion along a field line with local density increase expanding at the ion sound speed, temperature decrease modified by the electron heat flux along the field line, and resulting local pressure increase.

FIG. 2. Heat flux deposited on the wall near the midplane injection port on DIII-D, for a pellet triggered ELM (Mid Pellet), a natural ELM, for a pellet that did not trigger and ELM (No ELM), and for an X-point pellet (R-2 Pellet) that triggered an ELM. The Z coordinate is the distance above the injection port at that toroidal location.

FIG. 3. Plot of pellet ELM triggering statistics as a function of pellet speed and size for (a) JET-ILW from Ref. [14] and (b) DIII-D.

FIG. 4. Heat flux profile from DIII-D for the outer divertor strike point for a large natural ELM, pellet triggered ELM multiplied by a factor of 7.5 and inter-ELM heat flux multiplied by a factor of 15 . 
FIG. 5. ELM frequency needed on ITER as a function of plasma current to mitigate melting of the divertor and to eliminate the influx of $\mathrm{W}$ from the divertor from Ref. [2]. The expected natural ELM frequency is also shown.

FIG. 6. A comparison of a LFS triggered ELM and HFS fueling pellet ELM in the same plasma discharge on DIII-D. The midplane pellet light emission, magnetic loop signal, and the central and edge line integrated density $\left(\mathrm{cm}^{-2}\right)$ are shown. 

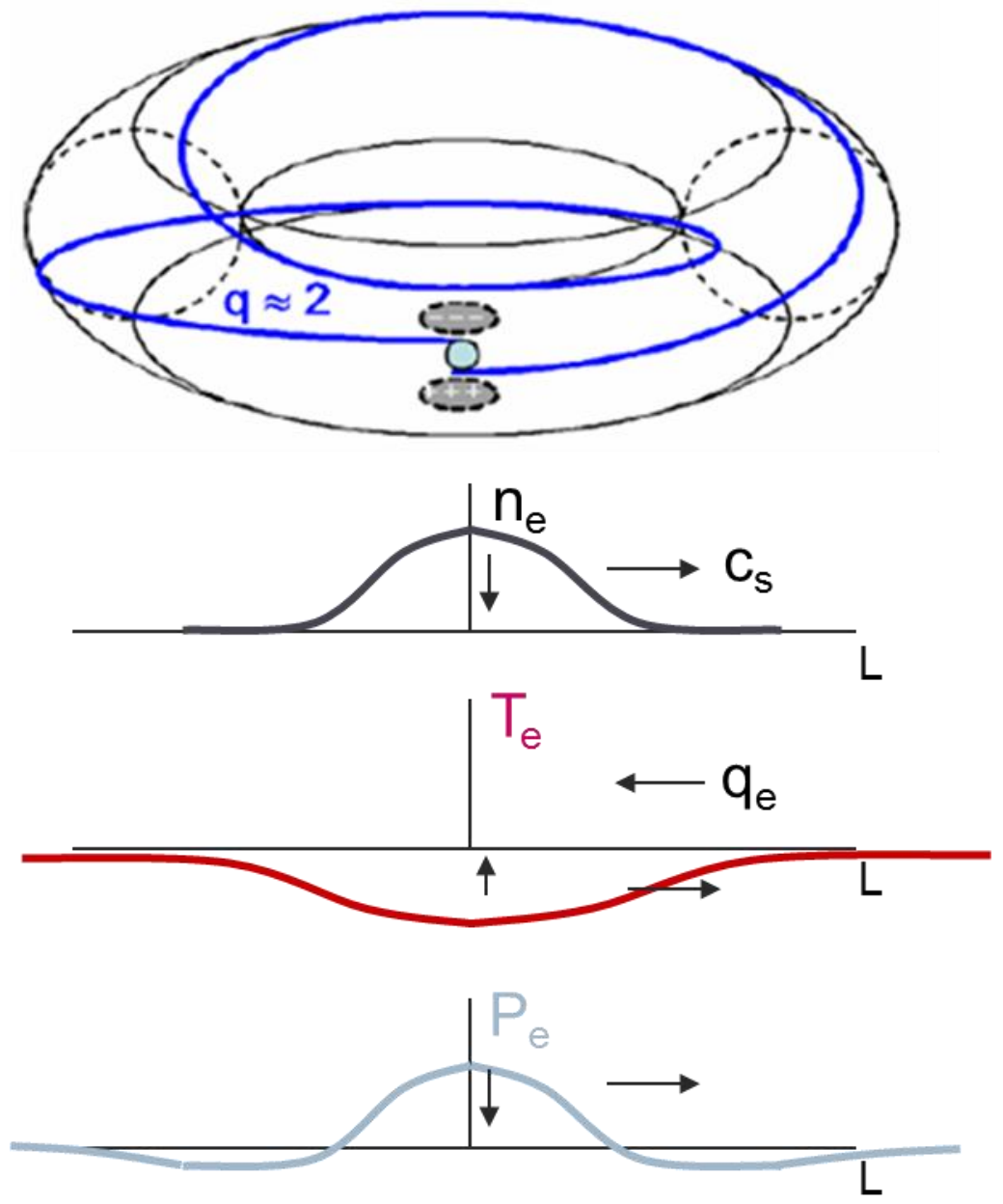

Fig. 1. 


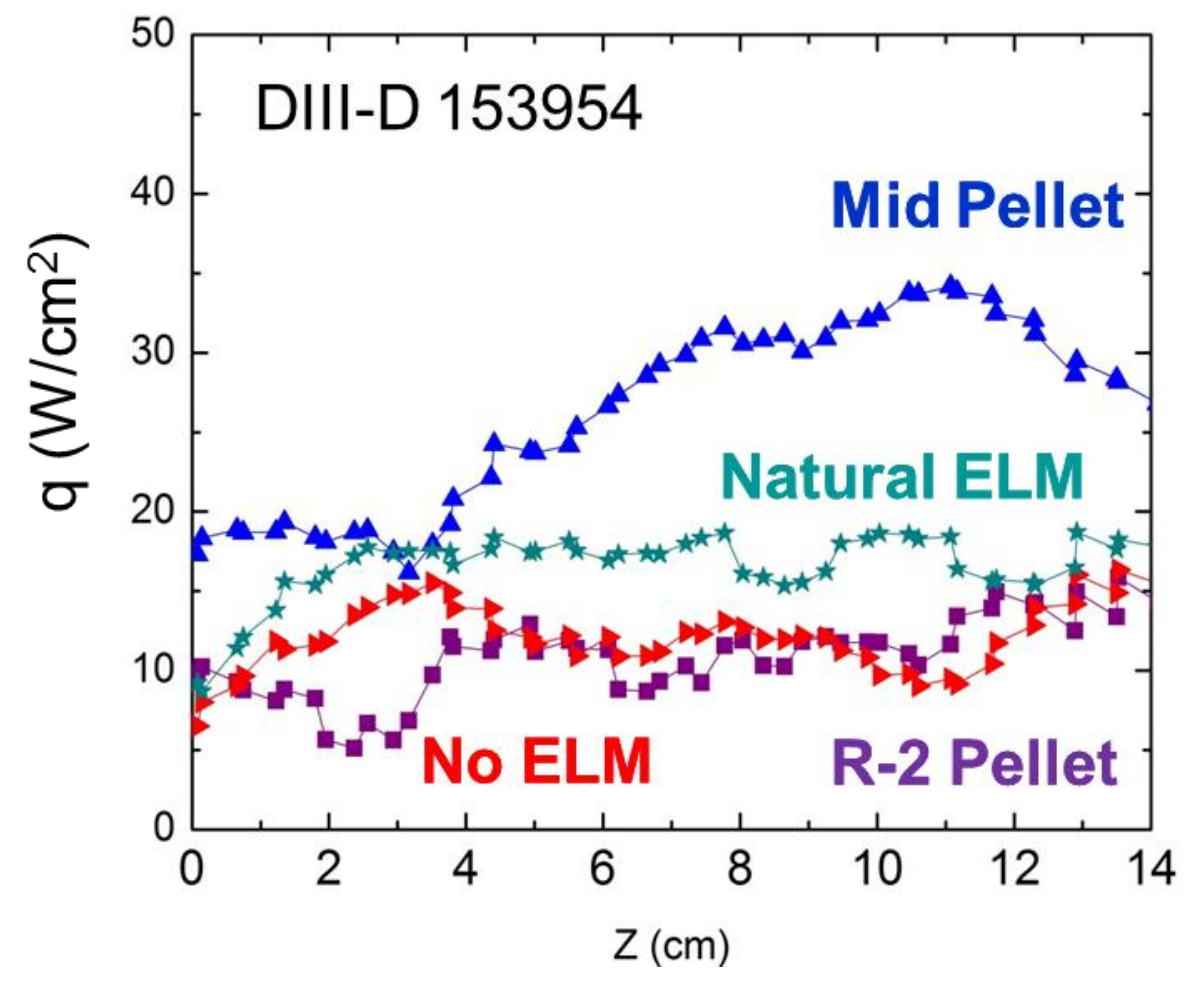

Fig. 2 


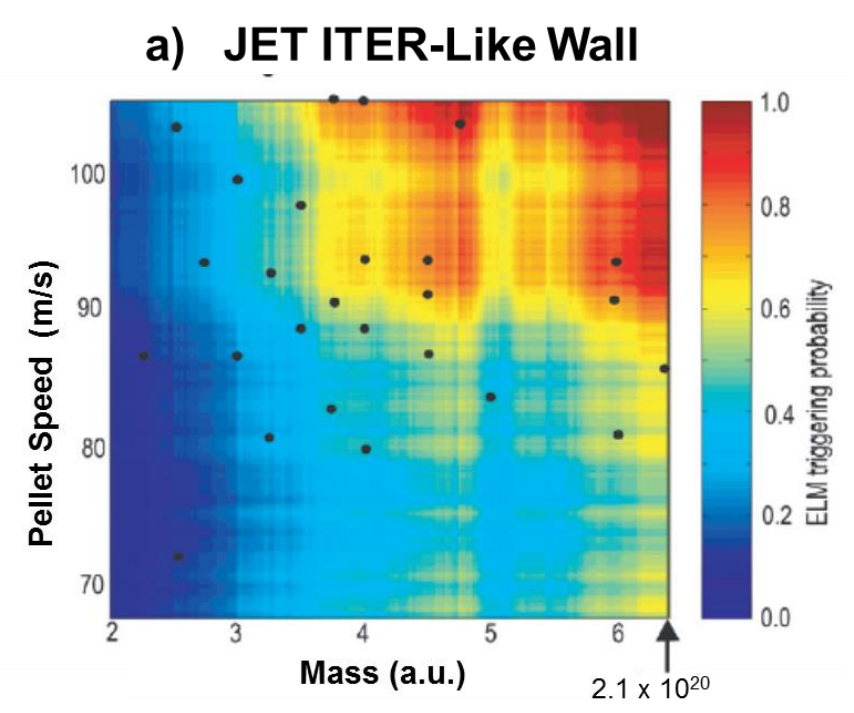

b) DIII-D ITER-Like Scenario

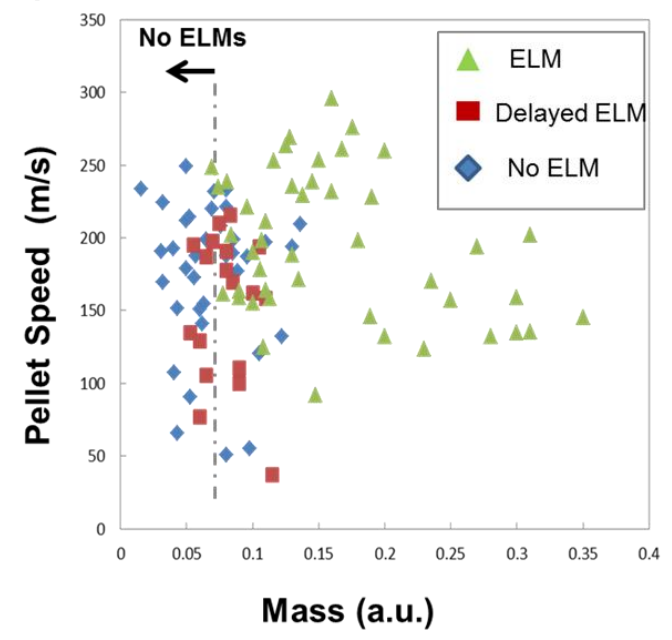

Fig. 3 


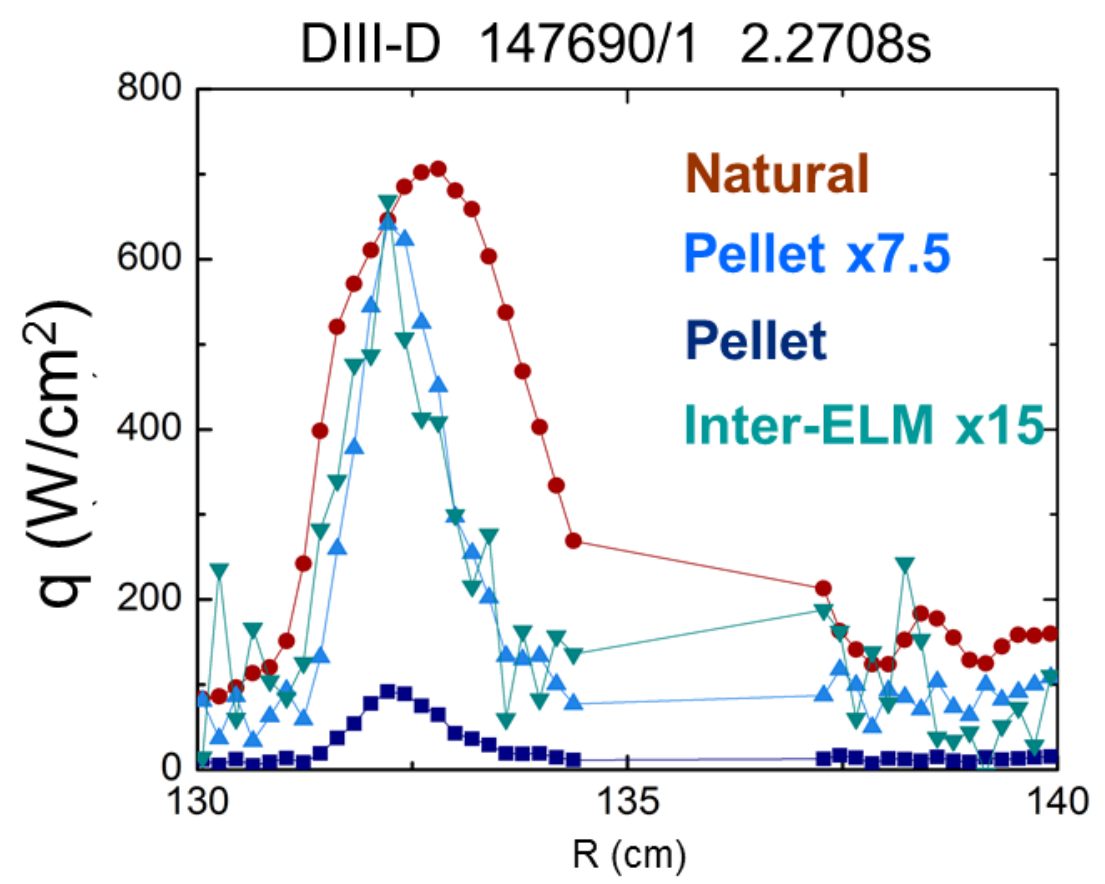

Fig. 4 


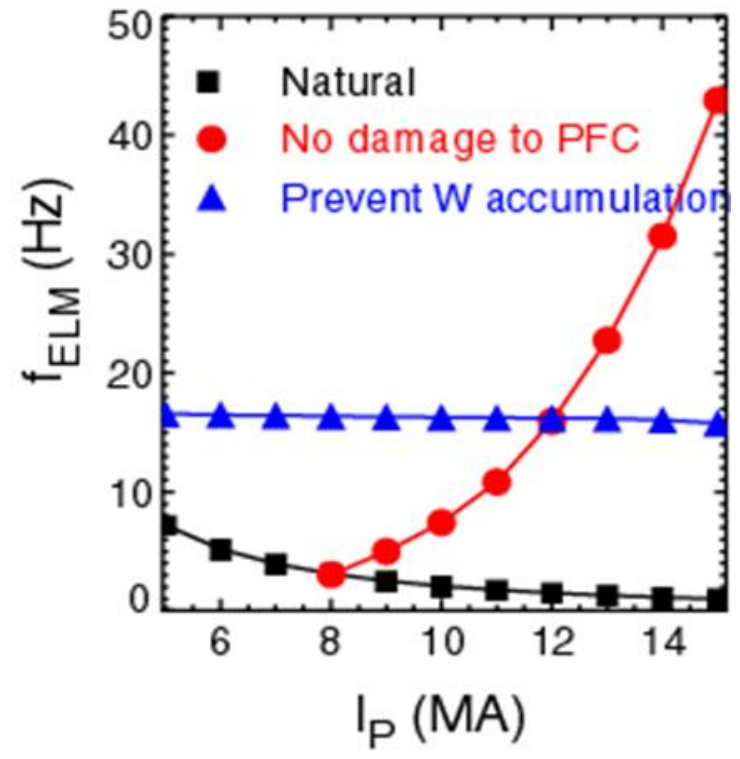

Fig. 5 


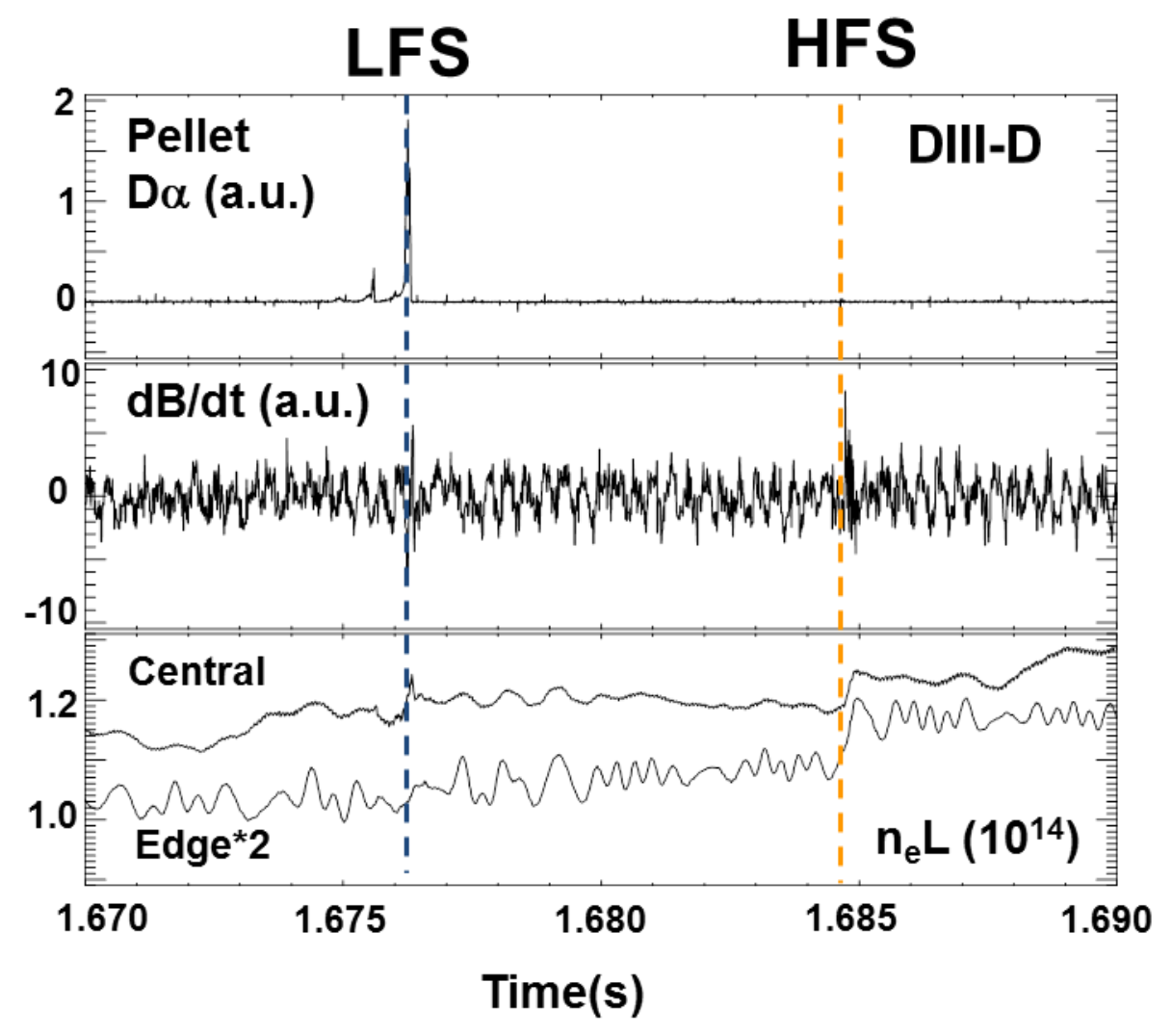

Fig. 6 